\title{
Post-senderismo, meta-seguridad y meta-violencia peruana en el caso VRAEM
}

\section{Post-Senderismo, Meta-Security and Peruvian Meta-Violence in the VRAEM Case}

\author{
César Niño ${ }^{1}$ \\ Universidad Sergio Arboleda (Colombia)
}

Recibido: 15-07-19

Aprobado: 29-10-19

\section{Resumen}

El presente artículo tiene como principal propósito establecer las categorías conceptuales de la meta-seguridad y la meta-violencia aplicadas al caso del VRAEM en Perú después del fin del Sendero Luminoso. Para cumplir con lo anterior, la pregunta a resolver a lo largo del artículo es ¿por qué la región del VRAEM se configura como un espacio en el que el senderismo ha mutado y representa una amenaza para los operadores de seguridad peruanos? El trabajo integra perspectivas teóricas y empíricas alrededor de la mutación y transformación de la violencia y la seguridad especialmente en la región del Valle de los ríos Apurímac, Ene y Mantaro.

Palabras-clave: post-senderismo, meta-seguridad, meta-violencia, VRAEM, Perú.

\begin{abstract}
The main purpose of this article is to propose the conceptual categories of meta-security and meta-violence applied to the case of VRAEM in Peru, after the end of the Sendero Luminoso. To accomplish the above, this paper

\footnotetext{
${ }^{1}$ (cesar.nino@usa.edu.co) Doctor en Derecho Internacional por la Universidad Alfonso X el Sabio (España). Director de Investigación y Profesor Asociado de la Escuela de Política y Relaciones Internacionales de la Universidad Sergio Arboleda (Colombia). Grupo de Análisis Político GAP. Sus últimas publicaciones son: "La construcción de los conceptos de la "sur-alternidad" y la geopolítica en el Caribe". Finisterra, 111, 2019, pp. 119-132 y "Colombia undergoing mutation: from the concept of post-conflict to the pragmatism of the conflict". JANUS.NET e-journal of International Relations, 9(2), 2018, pp. 78-92.

ORCID: https://orcid.org/0000-0002-1417-6643.
} 
is expected to answer the following question: Why the VRAEM region is constituted as a space in which trekking has mutated and represents a threat for the Peruvian security operators? This research incorporates theoretical and empirical perspectives around the mutation and transformation of violence and security, especially in the region of the Apurímac, Ene and Mantaro Valley.

Key-words: Post-Senderismo, Meta-Security, Meta-Violence, VRAEM, Peru.

\section{Introducción}

El presente trabajo tiene como propósito presentar reflexiones convergentes alrededor del post-senderismo, la seguridad y la violencia en Perú que se encuentran directamente ligadas a la región del Valle de los ríos Apurímac, Ene y Mantaro (VRAEM). Con el declive de la acción subversiva entre los años 1993 y 2000, la mutación del conflicto abrió dimensiones de reducción militar a combatientes senderistas, sometimiento a la justicia y en otros casos a la atomización y posterior focalización de los reductos en la zona del VRAEM. A inicios del siglo XXI (2001 en adelante), el fin del Sendero Luminoso como actor protagónico del conflicto en Perú trajo consigo derivaciones naturales en la transformación de la violencia y de escenarios en transición que representarían desafíos institucionales para la integridad y seguridad nacional.

Con base en lo anterior, en materia de conflictos irregulares, la tendencia es a que luego de la terminación de éste haya nuevas manifestaciones de violencia y se incrementen dinámicas rivalizantes que van en contravía de la seguridad del Estado (Collier, Hoeffler, y Söderbom, 2008). En efecto el VRAEM posee características vitales para la coexistencia de variables como el narcotráfico, plantaciones de cultivos ilegales, grupos armados, desnutrición infantil, mercados de la violencia, terrorismo, ausencia y precariedad estatal, entre otras. Aquellas variables representan un cúmulo estratégico para entender las razones del postsenderismo en la actividad criminal, la meta-seguridad y la meta-violencia conforme a las interpretaciones que representa la amenaza para el Estado peruano.

En efecto, la bibliografía existente sobre conflictos irregulares redunda en las explicaciones causales y fenomenológicas alrededor de los orígenes, interpretaciones discursivas de los enfrentamientos armados que generaron espirales de violencias y transformaciones institucionales sobre la base de la resolución de los conflictos, no obstante, las ideas sobre el análisis de los fenómenos de violencia y seguridad después de escenarios hostiles sigue en constante construcción alrededor de distintos casos de estudio como en su momento Bosnia (Campbell, 1998), Ruanda (Uvin, 2001), Sierra Leona (Mackenzie, 2009), Myanmar y su violenta transición a la democracia 
(Christensen, Nguyen, y Sexton, 2019) El Salvador (Del Castillo, 2001) o Colombia (Franco, Oliveira, y Ali, n.d.; Niño y Palma, 2017; Ríos y Gago, 2018). Por eso, una de las motivaciones principales del presente documento es mostrar un análisis sobre las implicaciones de la meta-violencia y la metaseguridad que se encuentra delimitada en un contexto post-senderista.

Así, es menester abordar al VRAEM como caso particular en Perú y la constante de grupos derivados del Sendero Luminoso en la configuración de un escenario explicativo sobre las nuevas presentaciones del crimen y violencia como amenaza para la seguridad nacional peruana.

De tal manera el trabajo se presenta en apartados que fungirán como estructura del documento. En primer lugar, se aborda una revisión de literatura relevante y se discutirá en torno a lo estudiado por distintos autores conforme al post-senderismo y la vinculación sobre los conceptos de metaseguridad y meta-violencia aplicados al caso peruano. Allí es importante la dimensión académica sobre la propuesta conceptual convergente alrededor del caso de estudio del VRAEM. Posteriormente se presentan las características metodológicas de la investigación haciendo énfasis en las principales aportaciones teóricas al caso de estudio, las reflexiones sobre la consecución de la información, las cifras y los datos relevantes que permitan la construcción analítica del documento acompañado de herramientas como entrevistas a profundidad y fuentes oficiales especializadas. Después se aborda la dimensión concerniente a la mutación criminal, la relación con la ausencia estatal en la zona del VRAEM y la complejidad geopolítica; a continuación, se generan reflexiones sobre la meta-violencia, el terrorismo y el post-senderismo en el VRAEM. Finalmente, las conclusiones que se presentan hacen parte de los análisis necesarios en la propuesta e innovación sobre la aproximación conceptual expuesta en el documento en escenarios de post-senderismo y de la meta-seguridad en Perú.

\section{Marco teórico y estado de la cuestión}

Los estudios sobre la violencia en Perú alrededor del protagonismo del Sendero Luminoso reposan en fuentes propiamente peruanas, estadounidenses o británicas (Ríos, 2018). Un diagnóstico que responde a lógicas de desconocimiento regional sobre la realidad latinoamericana y que constituye un asidero de críticas sobre lo construido de manera epistemológica al caso peruano (Prozorov, 2015). En otras palabras, la investigación del conflicto armado peruano ha sido hecha, en su gran mayoría, por autores extraregionales. En esencia la literatura en América Latina descansa en mayor dimensión sobre realidades no peruanas como reflejo de arquetipos singulares de la violencia 
marcada en los casos más representativos de El Salvador, Colombia, Nicaragua, Guatemala y Honduras.

No obstante, los trabajos que han reflejado la preocupación sobre las causas, problemáticas y transformaciones del conflicto en Perú corresponden a aproximaciones casuísticas focalizadas como los casos de Chuschi y Quispillaccta en Sánchez (2007), el conflicto agrario en la zona andina en Rénique (2004), los movimientos campesinos en Kápsoli y Valladares (1977), la génesis de un movimiento armado rural en McClintock (1984) la visión maoísta sobre la formación de Abimael Guzmán Reynoso en la proyección rural y empobrecida entre 1980 en el trabajo de Palmer (2014). Así mismo, existen investigaciones puntuales sobre el alcance estratégico del Sendero Luminoso en sus operaciones y tácticas de terrorismo (Beggar, 2005; Sullivan y Beittel, 2016) que a pesar de tener un número de integrantes no tan amplio y representativo, sometió las políticas de seguridad nacional con implicaciones de éxito para el grupo en su propia supervivencia (Berg, 1986; Favre, 1984) y a la reconstrucción desde el inicio, el desarrollo, el auge y el colapso de uno de los grupos armados más violentos de la historia reciente de América Latina (Ríos y Sánchez, 2018).

Por otra parte, trabajos basados en el posconflicto peruano versan sobre las condiciones del esclarecimiento y construcción de memoria que estuvieron limitadas a una versión oficial de las víctimas como lo menciona Macher (2014), también alrededor del seguimiento y cumplimiento de lo acordado sobre la no repetición por un reconocimiento de conflicto armado tardío (2003) que trae implicaciones sobre las responsabilidades judiciales y políticas (Gurmendi, 2017). De igual manera, los estudios sobre posconflicto en Perú hacen hincapié en las dificultades que ha traído la noción de no recordar lo ocurrido para construir un escenario de desarrollo nacional y que aquello trae problemas estructurales con miras al futuro peruano (Orihuela, 2012). La anterior radiografía bibliográfica responde a que si bien ha habido un interés por el caso peruano en términos académicos, las condiciones sobre la mutación de la violencia y particularmente sobre el reacomodamiento de facciones del Sendero Luminoso, se limitan a responder lógicas sobre la historia del grupo, las influencias ideológicas, y las metodologías de orden militar en la doctrina contraterrorista del Estado peruano, pero alejada de las reflexiones postsenderistas y de la meta-seguridad en Perú.

Sobre el post-senderismo la literatura se refiere al movimiento o facción denominada "Proseguir" (Santillán, 2017) que tiene que ver en esencia con los procesos violentos de transformación y adaptación de los residuos del grupo a partir del siglo XXI. Aquel evento después del clásico Sendero Luminoso, es la construcción de un grupo derivado que responde a lógicas alternativas de la tradicional visión guerrillera peruana. De igual manera, la existencia de un 
post-senderismo, a pesar de tener una rudimentaria bibliografía que se preocupe por el desenvolvimiento y explicación de la adaptabilidad del grupo, se debe a una ausencia y debilidad estatal que se traduce en la generación de dinámicas paraestatales enclavadas en territorio peruano. Al parecer esta dimensión es el común denominador en países de la región (O’Donnell y Wolfson, 1993) como Colombia, El Salvador, Honduras, Guatemala, México, entre otros.

Para efectos del presente artículo, el post-senderismo se entiende como la mutación del grupo sobre un bastión geográfico que alcanza a redefinir la noción de la seguridad en el Estado peruano, es decir, hay una reformulación del post-senderismo en dimensiones de adaptabilidad a los nuevos escenarios y de construcción de una meta-seguridad desde el Estado. Con base en lo anterior, las dimensiones tratadas sobre la meta-seguridad aplicada al caso peruano tienen un centro de gravedad en una seguridad multifacética que se constriñe a una dimensión puntual y a un lugar determinado, en esta oportunidad al VRAEM. En ese orden de ideas, los conceptos de meta-seguridad y meta-violencia no han sido explorados ni operacionalizados dentro de los estudios de conflicto ni de seguridad con gran calado. No obstante, las aproximaciones más profundas alrededor de la meta-violencia consisten en la construcción de antagonismos sociales (Saramo, 2017) que han sido producto de transformaciones que sobrepasan los clásicos esquemas de practicar la violencia.

Es un concepto que no se encuentra con gran facilidad en los trabajos sobre la violencia, sin embargo, autoras como Saramo (2017) sostiene que es un fenómeno directamente ligado al carácter emocional y es un punto de convergencia directo con el término de "violencia lenta" de Nixon (2011) que atribuye elementos estructurales de escenarios de conflicto con los de posconflicto. Si bien la meta-violencia suele ser compleja para ponerla en evidencia, en el contexto peruano del VRAEM es menester construir las suposiciones convencionales sobre la violencia como un acto altamente visible y digno de mención porque se centra en los eventos, el tiempo y el caso en el escenario de Perú. La meta-violencia se traduce en la relación que puede generar la noción entre los nuevos enclaves de la violencia senderista en dimensiones geográficas post-senderistas y en la arquitectura de una violencia ejercida por élites subterráneas, paralelas y asimétricas (Christensen et al., 2019).

Por otro lado, en materia de meta-seguridad, la operacionalización del concepto no existe en los albores de la literatura contemporánea. No obstante, trabajos como los de Niño y Palma (2017) se concentran en el concepto de "pos-seguridad" bajo el cual se advierte que un estado óptimo de seguridad no es directamente proporcional a un escenario sin violencia (Niño y Palma, 2017), es decir, la definición de la seguridad en el contexto de Perú se desprende de la clásica arquitectura rival con el Sendero Luminoso como objeto referente. Con base en lo anterior, en la necesidad de (re)pensar los estadios de seguridad 
de Perú en el VRAEM sumado a la meta-violencia que supone los derivados senderistas, se alcanza a poner en duda la dinámica de opuestos entre Estado peruano y grupos ilegales encaminado a la construcción de un enemigo absoluto a destruir para configurar las respuestas estatales en esta materia.

\section{Metodología de la investigación}

La pregunta de investigación sobre la que se construye el presente artículo es ¿por qué la región del VRAEM se configura como un espacio en el que el senderismo ha mutado y representa una amenaza para los operadores de seguridad peruanos? Bajo el anterior cuestionamiento, la hipótesis planteada responde que en el VRAEM se encuentran dimensiones de la meta-seguridad, meta-violencia, las capacidades criminales de los derivados del Sendero Luminoso y una compleja sistematicidad de ausencia estatal que permiten la aparición de espacios vacíos que finalmente son ocupados por actores ilegales con alta capacidad de fuego y control territorial, es decir, el VRAEM se alcana a configurar como un paraestado (O’Donnell y Wolfson, 1993).

Para construir el abordaje metodológico, en primer lugar, es necesario formular un análisis desde aproximaciones geopolíticas del VRAEM, se emplean caracterizaciones sobre la mutación criminal, el narcotráfico y la relación existente con la ausencia del Estado (Mendoza y Leyva, 2017). En ese orden de ideas, la información será extraída de fuentes oficiales peruanas como el Instituto Nacional de Estadística e Informática (INEI) recursos desclasificados de inteligencia peruana, fuentes académicas y periodísticas en aras del respectivo análisis. Posteriormente, las cuestiones relacionadas con las convergencias de actividades ilegales y la supervivencia de los residuos post-senderistas, son tratadas desde la óptica del estudio de caso valiéndose de entrevistas a actores relevantes en el entendimiento del contexto peruano, exmilitares, tomadores de decisiones y arquitectos de política pública que fungen en dicha región peruana.

Lo anterior será operacionalizado bajo los lentes conceptuales y teóricos propuestos para el desarrollo del presente artículo que responden a las dinámicas propias de lo formulado por McClintock (1984) en la explicación fenomenológica sobre la construcción del Sendero Luminoso en el VRAEM y la relación contemporánea con el narcotráfico, la ilegalidad y el terrorismo (Calmet y Salazar, 2013; Ríos, 2017, 2018; Santiago y Annoni, 2017). 


\section{Entre la mutación criminal y la ausencia estatal: una aproximación geopolítica compleja}

A pesar de existir conceso sobre los territorios que conforman al VRAEM ${ }^{2}$, geopolíticamente hay imprecisiones físicas que se mezclan simbióticamente con las fuerzas post-senderistas y con el avance de la hoja de coca como factor fundamental que ha logrado definir la región (Díaz, 2015). La cuestión geopolítica del VRAEM se configura como un caso relativo a la geopolítica crítica y alejada de los postulados tradicionales (Atkinson y Dodds, 2000; Dodds, Kuus, y Sharp, n.d.; Kuus, 2018; Slater, 2004). Es decir, es una zona que se distancia de los clásicos modelos eurocéntricos determinados por elementos físicos y de expansión territorial rivalizante con otros Estados, en esta oportunidad, aquella zona peruana es la manifestación que involucra factores domésticos, poblacionales, características de la agricultura, elementos criminales, de enclaves de conflicto y actores asimétricos como los postsenderistas.

En la lógica de la meta-violencia y meta-seguridad en Perú, el VRAEM parece encontrar un espacio único en el análisis geográfico y geopolítico en el territorio, no obstante, sus características físicas y de productividad son el común denominador de buena parte de zonas de América Latina. En ese sentido, aquella zona posee una economía esencialmente agropecuaria (en términos convencionales), poco diversificada y dominada por el cultivo de la hoja de coca (Mendoza y Leyva, 2017, p. 13). En términos físicos, dicha región comprende 50 distritos y una superficie territorial de aproximadamente $58.163,4$ kilómetros cuadrados. Una zona compleja en la cual se encuentran el departamento de Apurímac, Ayacucho, Cusco, Huancavelica y Junín. El VRAEM parece poseer características geográficas plausibles para el sistema agropecuario pero la realidad corresponde a que tiene 1.9 parcelas por unidad agropecuaria, una cifra que se ubica por debajo del promedio nacional peruano de 2.2 (INEI, 2013). El problema responde al minifundismo y la presencia dominante del tráfico ilícito de drogas que distorsiona la economía por el blanqueo de los recursos financieros provenientes del narcotráfico (INEI, 2013, p. 1). En ese sentido, mientras el café o el cacao que se producen en el VRAEM le da una cosecha al año a la región, la hoja de coca les da 3 y a veces 4 cosechas anuales, y con una rentabilidad muy superior (Iglesias, 2012). Así se alcanzan a explicar las razones sobre la atracción que genera en términos económicos y geográficos la producción de coca que desemboca en productos ilícitos.

El VRAEM tiene una superficie de coca en producción de 20,304 hectáreas y representa el 46\% de la coca total de Perú (UNODC, 2017). En efecto, la totalidad de la producción de coca en esta zona se articula y mezcla

\footnotetext{
${ }^{2}$ Bajo la narrativa oficial de Perú, el río Amazonas nace en el VRAEM (INEI, 2013).
} 
con el narcotráfico para la generación de base y clorhidrato de cocaína que se comercializan a través de rutas aéreas clandestinas y por medio de vías alternas (Santiago y Annoni, 2017). Para mitigar este fenómeno el Estado emitió la Ley 30339 de Control, Vigilancia y Defensa del Espacio Aéreo Nacional, que permite la interceptación e interdicción en vuelo para evitar el tráfico de drogas (UNODC, 2017). Con aquel instrumento jurídico pareciera en principio que el Estado intenta generar institucionalidad en una zona convulsa y altamente compleja, no obstante, las asimetrías de la zona permiten que el Estado sea extranjero en su propio territorio y las economías ilegales alcancen umbrales de existencia que ponen en jaque la seguridad nacional peruana.

Bajo la lógica de aproximación geopolítica y el hallazgo convergente alrededor de la criminalidad, para Díaz (2015) vale la pena tener en cuenta algunas variables fundamentales. En primer lugar, la ubicación geográfica es una variable problemática al tener una configuración indeterminada, imprecisa, irregular y difusa (Díaz, 2015; INEI, 2013). Inclusive, las propias versiones oficiales del Estado peruano con sus distintas instituciones se contradicen sin cartografías unificadas y homogéneas (Calmet y Salazar, 2013; Díaz, 2015, p. 50). Lo anterior se convierte en un problema para los tomadores de decisiones y arquitectos de las políticas de seguridad al encontrar ambigüedades cartográficas y al mismo tiempo una oportunidad para los grupos ilegales al entender los espacios vacíos que el propio Estado ha generado. Partiendo de lo anterior, el VRAEM tiene características físicas de difícil acceso por la dinámica de la naturaleza que lo compone, de tal manera, el tránsito para el acceso a la zona es complejo, accidentado y agreste (Díaz, 2015; Mendoza y Leyva, 2017) por lo que el control territorial por parte del Estado se convierte en un reto estratégico de gran envergadura, pero para los grupos ilegales es el mejor ecosistema para subsistir.

La segunda variable es la población. Esta dimensión en el VRAEM corresponde a que las características poblacionales oscilan en rangos etarios altamente productivos (INEI, 2019). En efecto es una zona compleja y heterogénea no solo geográficamente sino poblacionalmente, de tal manera cohabitan poblaciones rurales y urbanas que chocan en conflictos sociales y económicos. La población urbana sobresale en número sobre la rural por el desplazamiento de comunidades a causa de la violencia motivando a grandes movilidades humanas hacia las ciudades y dejando espacios vacíos rurales (Díaz, 2015, p. 60). Como tercera variable y enlazado con la anterior, la cuestión socioeconómica representa una situación relevante. Los jóvenes entre 14 y 19 años de edad abandonan la educación básica regular en la secundaria, presumiblemente para dedicarse a la cosecha y producción de la hoja de coca. Así las cosas, se estima que 40 de cada 100 jóvenes entre 14 y 18 años de edad en el VRAEM no están estudiando. Sumado a lo anterior, el 90\% de 
los pobladores se dedican a actividades asociadas al cultivo de la coca por la alta rentabilidad derivada del trabajo en esa actividad ilícita (Lavado, 2013, p. 136). Las condiciones permiten que las economías ilegales y la ausencia estatal construyan fenómenos que ponen en riesgo la seguridad nacional de Perú. Vinculado a lo anterior, la pobreza en el valle corresponde al $76.1 \%$ de la población y la pobreza extrema alcanza a la mitad $(50,1 \%)$ del VRAEM que corresponde al 13,7 \% a nivel nacional (Comando Conjunto de las Fuerzas Armadas, 2017).

En cuarto lugar, el tráfico de drogas se suma a las variables que hacen del VRAEM un escenario complejo y de gran interés estratégico criminal en la era post-senderista. El VRAEM es el valle cocalero de Perú (Díaz, 2015; Santillán, 2017) en el que las rutas, los actores, los agroquímicos, la tecnificación y los espacios vacíos representan un teatro altamente confuso para luchar contra las drogas. Las condiciones indican que el desarrollo cocalero, las facciones post-senderistas (Díaz, 2015; UNODC, 2017), la meta-violencia y la ausencia del Estado tienen correlación. Finalmente, el terrorismo se configura como una variable crucial al entender el complejo entramado del VRAEM. A saber, los remanentes del Sendero Luminoso, el narcotráfico, las naturalezas poblacionales, la accidentalidad geográfica y la ausencia estatal producen un escenario propicio para que el valle sea un lugar de convergencias de la meta-violencia en Perú (Ríos, 2019). En efecto los sobrevivientes al antiguo grupo de Guzmán se reagruparon para responder estratégica y tácticamente a un ambiente complejo, centrando sus relaciones con la población civil, en particular con los pequeños agricultores productores de coca (Taylor, 2017). A pesar de que el ejército peruano se encuentre en la zona, en particular el Comando Especial-VRAEM, el Estado sigue ausente en la región, es decir, la calidad de la presencia de las instituciones no se mide por el número de instituciones físicas allí presentes (Dahl, 1999; O’Donnell y Wolfson, 1993).

\section{Meta-violencia, terrorismo y post-senderismo en el VRAEM}

Los remanentes del Sendero Luminoso alcanzaron un nivel de adaptabilidad y de cambio entre sus operaciones clásicas en zonas urbanas y de sierra a lógicas selváticas en el VRAEM. Las cuestiones geográficas cambian drásticamente las metodologías de violencia en el teatro irregular. Entre 2001 y 2003 luego de un ataque a la policía en Ayacucho, el presidente Alejandro Toledo (2001-2006) determinó una guerra contra el terrorismo. A raíz del periodo post-fujimorista, el ejército tenía restricciones para operar en las dinámicas domésticas en Perú, cuestión que produjo una baja capacidad estratégica y un obsoleto sistema de lucha contrainsurgente. De tal manera, los 
primeros ataques en el VRAEM fueron sobre la policía y un secuestro masivo a 71 funcionarios de la compañía argentina de ingenieros Techint (Ariza, 2019; La Nación, 2003; Misión permanente de Perú ante la ONU, 2005).

Si bien hubo restricciones operativas del ejército para hacer frente al terrorismo, las políticas de seguridad en Perú enfocadas a la lucha contraterrorista tuvieron construcciones doctrinales desde el 2006 enfocadas directamente sobre el VRAEM. En esa medida, bajo la presidencia del Alan García (2006-2011) en el 2007 se declara la intervención y presencia estratégica en la zona como de interés nacional, siendo las fuerzas armadas la única institución capaz de ejercer presencia en el valle (Calmet y Salazar, 2013). Aquella disposición castrense se denominó inicialmente Plan VRAE y produjo una declaratoria de emergencia por 60 días en la zona a raíz de varios ataques subversivos, de manera sucesiva, la securitización de la región alcanzó a materializarse bajo lógicas exclusivamente militaristas (Berg, 1986; Calmet y Salazar, 2013; Comando Conjunto de las Fuerzas Armadas, 2017; Gurmendi, 2017). Por tal motivo, aquellas políticas brindaron espacios plausibles para la generación de brechas sociales, estratégicas y de conflicto asimétrico. Lo anterior alcanzó a generar problemáticas asociadas a los derechos humanos y no réditos estratégicos en la lucha contra el terrorismo post-senderista.

Por lo tanto, la sofisticación y tecnificación criminal redunda en las lógicas de un marco de enclave senderista. Los grupos que alcanzan a cohabitar en el VRAEM compiten por la existencia grupal rivalizada con los "otros", por las rutas del narcotráfico ${ }^{3}$, por el control de cultivos de coca, por las hegemonías sociales y poblacionales, por la ocupación territorial, la capacidad de fuego y por la invisibilidad que alcanzan en la permeabilidad de las estructuras campesinas y sociales. En otras palabras, los spin-off del Sendero Luminoso se valen de la violencia y conocimiento geográfico, así como de la experiencia adquirida en términos criminales para brindar seguridad a sus economías ilegales y comercio subterráneo.

La meta-violencia en el VRAEM se construye a partir de elementos definitorios bajo los herederos senderistas (Ríos, 2017). El final del clásico Sendero Luminoso motivó a una atomización y derivación del extinto grupo en subgrupos, células y corpúsculos como el Proseguir o Sendero Rojo (Ríos, 2018; Santillán, 2017), así mismo, las crisis internas frente a las posiciones políticas, ideológicas y reformativas motivaron a las subdivisiones de los anteriores grupos, en otras palabras, la captura de Abimael Guzmán en la Operación Victoria de 1992 desató una ruptura interna, organizacional y de identidad revolucionaria. Sin embargo, unos de los herederos más importantes de Guzmán son los hermanos Quispe Palomino, quienes han logrado reconstruir

\footnotetext{
${ }^{3}$ Vale la pena aclarar que según la UNODOC (2017) de la región del VRAEM sale la mayor parte de cocaína de Perú.
}

Araucaria. Revista Iberoamericana de Filosofia, Política, Humanidades y Relaciones Internacionales, año $22, \mathrm{n}^{\circ} 43$. Primer semestre de 2020. Pp. 349-365. ISSN 1575-6823 e-ISSN 2340-2199 https://dx.doi.org/10.12795/araucaria.2020.i43.18 
y tecnificar las formas de violencia y terrorismo en el valle a pesar de no reconocerse como sucesores de Guzmán y viceversa. Desde 1999 los hermanos Víctor y Jorge Quispe Palomino controlan los corpúsculos senderistas del VRAEM y han puesto en vilo la seguridad nacional desde la selva peruana.

Las economías ilegales sumadas al terrorismo son las principales características de acción de los actores irregulares que se encuentran en el valle. La cuestión de la meta-violencia radica en que no solo los herederos senderistas compiten por el control geográfico, económico y poblacional, también existen grupos de orden subterráneo que ponen en jaque las estrategias de seguridad del Estado. Algunos de estos grupos o clanes criminales tienen marcas y logos que identifican los cargamentos de droga como: delfin, escorpión, estrella, trébol, pingüino o las siglas $U F F, K W X, A M G, G O L D S$, el 99, la silueta de Machu Picchu, la letra $K$ o el mapa del Perú (Ariza, 2019). Los anteriores sellos corresponden a nuevos capos que han alcanzado niveles de industrialización criminal con alrededor de 200 individuos que los conforman, aquellos capos tienen por alias 'Viejo Guti', 'Comanche', Galleta', 'Huatari', 'Chipy' y 'Gato'(Comando Conjunto de las Fuerzas Armadas, 2017; La República, 2018).

La meta-violencia en el VRAEM recrea las complejidades estratégicas que el Estado peruano enfrenta. Por un lado, las dificultades operacionales de las fuerzas de seguridad para hacer control territorial efectivo se imposibilitan por la incapacidad e insostenibilidad de la política pública en una geografía agreste que involucra actores irregulares con alta capacidad de control y fuego. Por el otro, la dinámica irregular y asimétrica del VRAEM representa una amenaza para la seguridad nacional de Perú (Barnett, 2010; La República, 2018; Ríos, 2017). La configuración de escenarios que alcanzan a poner en riesgo la existencia y funcionamiento de las instituciones, representan lógicas clasificadas como amenazas a la seguridad (Buzan, Wæver, y Wilde, 1998), es decir, los grupos irregulares que se encuentran en el VRAEM tejen capacidades más allá de las criminales para reemplazar las funciones básicas del Estado, entre ellas el control del territorio.

De tal manera, los post-senderistas se han valido del terrorismo como metodología para socavar y hostigar a la población junto a las instituciones peruanas, en ese sentido, la eficacia de las fuerzas del Estado en el VRAEM es baja, las acciones terroristas en el valle representan un escenario adverso y concurrente en la frecuencia de actos por parte de Proseguir, el Movimiento por la Amnistía de los Derechos Fundamentales (MOVADEF) que, según estadísticas oficiales entre el 2006 y 2013 perpetraron una media de 100 atentados por año (INEI, 2013, 2019; Santillán, 2017). En efecto la triada post-senderismo, metaviolencia y terrorismo se convierten en elementos que resignifican la seguridad de Perú especialmente en el VRAEM, en otras palabras, la coexistencia de los elementos anteriormente mencionados imposibilitan un control efectivo del 
Estado en su territorio produciendo bastiones y resguardos estratégicos para los spin-off del senderismo que brindan una redefinición de futuras nuevas alianzas y facciones junto con otros grupúsculos narcotraficantes (Ríos, 2017) de Bolivia, Colombia, México, Serbia, Rusia y Afganistán especialmente.

Los anteriores actores irregulares tienen al Estado peruano como enemigo común, cuestión que aglutina la construcción de algunas confianzas estratégicas para la subsistencia a pesar de la competencia entre ellos. Las explicaciones alrededor de la capacidad operativa y de control territorial que tienen los distintos grupos armados que cohabitan en el VRAEM se sustentan por la simbiosis que existe entre los hermanos Quispe Palomino que cobran cupos a los demás grupos para exportar droga por vía aérea o terrestre y a cambio, hacen pistas clandestinas para avionetas, brindan seguridad a miembros de carteles y a los pasantes de los insumos para la pasta base de la cocaína (El Comercio, 2017; Iglesias, 2012). En otras palabras, aquella zona representa un complejo criminal industrial de gran envergadura.

De tal manera, la complejidad criminal, el terrorismo y la geográfica sumado al punto común de la coca, representan una de las explicaciones más robustas sobre el post-senderismo en el VRAEM. Así las cosas, es importante resaltar que la configuración de la amenaza post-senderista en aquella zona no es debido al número de efectivos sino a la capacidad de estos para socavar el territorio e impedir la presencia de las instituciones estatales. En materia de amenazas asimétricas e irregulares, la cuantificación de los actores es irrelevante cuando de capacidades se trata (Niño, 2017). En otras palabras, es interesante cómo reductos, derivados y post-grupos alcanzan a desestabilizar desde lo micro a lo macro, pues en palabras del expresidente peruano Fernando Belaunde Terry (1980-1985), "una bomba puesta por terroristas aparece en los titulares de la prensa mundial, pero una tonelada de dinamita detonada para construir una carretera no recibe ninguna notificación" (Bruce, 1984). La metaseguridad y meta-violencia en momentos post-senderistas son los conceptos que alcanzan a redefinir la situación de seguridad y violencia en el VRAEM.

Es importante resaltar que la violencia se convierte en un ejercicio cohesionador de alto valor estratégico para los post-senderistas. La metaviolencia construye escenarios complejos en los que los actores allí presentes intentan demostrar predominio violento bajo acciones terroristas. Por lo tanto, la construcción de meta-violencia es el producto de ejercicios de violencia por parte de élites subterráneas que, a pesar de enfrentarse a las acciones estatales, subsisten gracias las lógicas asimétricas, patrones alternativos y nociones de éxito distintas a las del Estado peruano. 


\section{Conclusión}

Los estudios e investigaciones que surgen luego de los conflictos armados, así como los marcos conceptuales alrededor de la seguridad en escenarios en constante transformación, presentan grandes derroteros sobre los avances en la descripción y clasificación académica en los asuntos que vienen luego de superados los conflictos. En otras palabras, la arquitectura terminológica puede ser un comienzo en la explicación de los fenómenos después de los escenarios traumáticos de los conflictos. De tal manera, el presente artículo pretendió formular y contribuir a los análisis complejos sobre la seguridad y la violencia en Perú, específicamente en el VRAEM conceptos capaces de integrar realidades dinámicas y particulares.

La situación del VRAEM en Perú es una muestra de la capacidad de mutación y transformación de la violencia y la seguridad en contextos asimétricos. La variable geográfica y las condiciones geopolíticas son características esenciales para un análisis robusto alrededor de los fenómenos allí dentro. Al parecer, la arquitectura bibliográfica y los referentes académicos han tenido objetivos comunes sobre la explicación del conflicto y la historia del Sendero Luminoso, así como las motivaciones ideológicas y los procesos de violencia dentro Perú; no obstante, se ha dejado de analizar de manera sistemática el escenario post-senderista salvo algunos estudios mencionados anteriormente. Partiendo de lo anterior, los enfoques que se han dado sobre el VRAEM han sido reducidos a dimensiones desde la criminalidad, por aquella razón, el presente artículo intenta girar la perspectiva y proponer términos aprovechando los vacíos conceptuales para ocupar las brechas analíticas sobre el fenómeno del VRAEM.

Lo anterior es apenas una propuesta que necesita mayores aportes y debates para abrir nuevas líneas de investigación sobre las cuales se construyan variables y enfoques precisos para las explicaciones más complejas sobre las realidades peruanas y por qué no, del resto de América Latina.

\section{Referencias bibliográficas:}

Ariza, J. (2019). Clanes y grupos criminales en el VRAEM, Perú. Entrevista. Atkinson, D., y Dodds, K. (2000). Geopolitical Traditions: A Century of Geopolitical Thought, 2000 | Online Research Library: Questia. Retrieved from https://www.questia.com/library/102763596/geopolitical-traditionsa-century-of-geopolitical. 
Barnett, K. (2010). El Resurgimiento de Sendero Luminoso (SL). Air y Space Power Journal.

Beggar, A. (2005). The path of state terror in Peru. In N. Menjívar, Cecilia y Rodríguez (Ed.), When states kill : Latin America, the U.S., and technologies of terror (1st ed., pp. 252-277). Austin: University of Texas Press.

Berg, R. (1986). Sendero Luminoso and the Peasantry of Andahuaylas. Journal of Interamerican Studies and World Affairs, 28(4), 165-196. https://doi. org/10.2307/165750

Bruce, R. (1984). Peru's Strange Shining Path. Retrieved February 24, 2019, from The Washington Post website: https://www.washingtonpost.com/ archive/politics/1984/01/31/perus-strange-shining-path/d806faca-e0024f8b-a918-8f42d7e705e5/?noredirect $=$ onyutm_term $=.7 \mathrm{e} 6 \mathrm{c} 7 \mathrm{a} 74190 \mathrm{~b}$

Buzan, B., Wæver, O., y Wilde, J. (1998). Security: a new framework for analysis. London: Lynne Rienner Pub.

Calmet, Y., y Salazar, D. (2013). VRAEM: Políticas de Seguridad Pública en Zona de conflicto. Cuadernos de Marte, (5), 157-186.

Campbell, D. (1998). MetaBosnia: narratives of the Bosnian War. Review of International Studies, 24(2), 261-281.

Christensen, D., Nguyen, M., y Sexton, R. (2019). Strategic Violence During Democratization. Evidence from Myanmar. In World Politics (Vol. 2). https://doi.org/10.1017/s0043887118000308

Collier, P., Hoeffler, A., y Söderbom, M. (2008). Post-Conflict Risks. Journal of Peace Research, 45(4), 461-478. https://doi. org/10.1177/0022343308091356

Comando Conjunto de las Fuerzas Armadas. (2017). Valle de los ríos Apurímac, Ene y Mantaro. Retrieved April 2, 2019, from http://www.ccffaa.mil.pe/ menuDEFENSA/vraem.htm

Dahl, R. (1999). La democracia : una guía para los ciudadanos. Madrid: Taurus.

Del Castillo, G. (2001). Post-conflict reconstruction and the challenge to international organizations: The case of El Salvador. World Development, 29(12), 1967-1985. https://doi.org/10.1016/S0305-750X(01)00090-0

Díaz, F. (2015). Por el camino del Neosenderismo...Crimen Organizado en el Perú: Neosenderismo en la Región del Valle del Río Apurímac y Ene. Universidad Nacional de La Plata.

Dodds, K., Kuus, M., y Sharp, J. (n.d.). Introduction: Geopolitics and its Critics. Retrieved May 24, 2018, from http://www.unice.fr/crookall-cours/ iup_geopoli/docs/Ashgate-Research-Companion-to-Critical-GeopoliticsIntro.pdf 
El Comercio. (2017). ¿Por qué no se ha logrado derrotar a Sendero Luminoso en el Vraem? | Política | El Comercio Perú. Retrieved February 24, 2019, from https://elcomercio.pe/politica/logrado-derrotar-sendero-luminosovraem-noticia-457606

Favre, H. (1984). Pérou : Sentier Lumineux et horizons obscurs. Problemes d'Amerique Latine, 72, 3-27.

Franco, I. B., Oliveira, J. A. P. De, y Ali, S. H. (n.d.). Peace with Hunger : Colombia's Checkered Experience with Post-Conflict Sustainable Community Development in Emerald-Mining Regions. 1-17. https://doi. org/10.3390/su10020504

Gurmendi, A. (2017). The Era of Terrorism: The Peruvian Armed Conflict and the Temporal Scope of Application of International Humanitarian Law. SSRN Electronic Journal. https://doi.org/10.2139/ssrn.2927155

Iglesias, I. (2012). Sendero Luminoso y el Narcotráfico en el VRAEM. Air y Space Power Journal, 24(4), 55-62.

INEI. (2013). Iv cenagro. Resultados Preliminares del Valle de los Ríos Apurímac, Ene y Mantaro - VRAEM a Nivel Distrital. Lima: Ministero de Agricultura.

INEI. (2019). ABC de la Estadística INEI. Retrieved April 1, 2019, from http://abc.inei.gob.pe/abc-de-la-estadistica/abc/un-dia-en-cifras/\#. XKLKjlUzbIU

Kápsoli, W., y Valladares, M. (1977). Los movimientos campesinos en el Perú, 1879-1965 - Wilfredo Kápsoli Escudero, Manuel Valladares - Google Libros (Delva). Lima.

Kuus, M. (2018). Critical Geopolitics. https://doi.org/10.1093/ acrefore/9780190846626.013.137

La Nación. (2003). Rescatan a los empleados de Techint. Retrieved April 8, 2019, from https://www.lanacion.com.ar/el-mundo/rescatan-a-losempleados-de-techint-nid502900

La República. (2018). Seis narcos controlan la mitad de toda la droga que sale del Vraem. Retrieved March 21, 2019, from https://arepublica.pe/ sociedad/1360033-seis-narcos-controlan-mitad-droga-sale-vraem

Lavado, P. (2013). Transición escuela- trabajo: Capacitación técnicoproductiva para escolares en el VRAEM. In Informe Final. Comisión Quipu para el $\operatorname{VRAEM}$ (p. 136). Lima: Ministerio de Desarrollo e Inclusión Social.

Macher, S. (2014). ¿Hemos avanzado?: a 10 años de las recomendaciones de la Comisión de la Verdad y la Reconciliación. Lima: Instituto de Estudios Peruanos IEP. 
Mackenzie, M. (2009). Security Studies Securitization and Desecuritization: Female Soldiers and the Reconstruction of Women in PostConflict Sierra Leone. Security Studies, 18(2), 241-261. https://doi. org/10.1080/09636410902900061

McClintock, C. (1984). Why Peasants Rebel: The Case of Peru's Sendero Luminoso. World Politics, 37(01), 48-84. https://doi.org/10.2307/2010306 Mendoza, W., y Leyva, J. (2017). La economía del VRAEM: diagnóstico y opciones de politica. Lima: USAID-CIES.

Misión permanente de Perú ante la ONU. (2005). Intervención del señor presidente de la República del Perú, Alejandro Toledo en el debate general. Retrieved from https://www.un.org/webcast/ga/60/statements/peru050918spa.pdf

Niño, C. (2017). El terrorismo como régimen internacional subterráneo: más allá de una lógica convencional. In Ediciones USTA. CO.

Niño, C., y Palma, D. (2017). Seguridad en Colombia: de la rigidez a la posseguridad. In J. Cubides Cárdenas y J. Jiménez Reina (Eds.), Desafios para la seguridad y defensa nacional de Colombia: teoría y praxis. https:// doi.org/10.25062/9789585625259

Nixon, R. (2011). Slow violence and the environmentalism of the poor. Cambridge: Harvard University Press.

O’Donnell, G., y Wolfson, L. (1993). Acerca del estado, la democratización y algunos problemas conceptuales: Una perspectiva latinoamericana con referencias a paises poscomunistas. Desarrollo Económico, 33(130), 163. https://doi.org/10.2307/3467251

Orihuela, J. (2012). Post-Conflict Economic Policy and Group Inequalities in Peru. In V. R. Langer A., Stewart F. (Ed.), Horizontal Inequalities and Post-Conflict Development (pp. 186-208). https://doi. org/10.1057/9780230348622_8

Palmer, D. S. (2014). The influence of Maoism in Peru. In A. C. Cook (Ed.), Mao's Little Red Book (pp. 130-146). https://doi.org/10.1017/ CBO9781107298576.009

Prozorov, S. (2015). La alteridad en las Relaciones Internacionales. Relaciones Internacionales , 29, 179-203. Retrieved from https:// repositorio.uam.es/xmlui/bitstream/handle/10486/677111/RI_29_10. pdf? sequence $=1$ yisAllowed $=\mathrm{y}$

Rénique, J. (2004). La batalla por Puno : conflicto agrario y nación en los Andes peruanos 1866-1995. Lima: IEP.

Ríos, J. (2017). Sendero Luminoso, 25 años después. Retrieved February 24, 2019, from Esglobal website: https://www.esglobal.org/senderoluminoso-25-anos-despues/

Ríos, J. (2018). Sendero Luminoso : Una apología de la violencia. Revista de Cultura de Paz, 2, 1-18. 
Ríos, J. (2019). Narratives about Political Violence and Reconciliation in Peru. Latin American Perspectives, 46(5), 44-58. https://doi. org/10.1177/0094582X19856890

Ríos, J., y Gago, E. (2018). Realidades y desafíos de la paz territorial en Colombia. Papers. Revista de Sociologia, 103(2), 281. https://doi. org/10.5565/rev/papers. 2361

Ríos, J., y Sánchez, M. (2018). Breve historia de Sendero Luminoso. Madrid: Catarata.

Sánchez, M. (2007). Pensar los senderos olvidados de historia y memoria : la violencia política en las comunidades de Chuschi y Quispillaccta, 19801991. Lima : Asociación Servicios Educativos Rurales, SER.

Santiago, D., y Annoni, D. (2017). El valle de los ríos Apurímac, Ene y Mantaro - Vraem: entre el narcoterrorismo y el descaso del estado peruano. Nuevo Derecho, 12(18), 107. https://doi.org/10.25057/2500672x.787

Santillán, P. (2017). Sendero Luminoso: evolución histórica y relevancia actual. Madrid.

Saramo, S. (2017). The Meta-violence of Trumpism. European Journal of American Studies, 12(2). https://doi.org/10.4000/ejas.12129

Slater, D. (2004). Geopolitics and the post-colonial : rethinking North-South relations (John Wiley y Sons, Ed.). Blackwell Publishing.

Sullivan, M. P., y Beittel, J. (2016). Latin America: Terrorism Issues. In Congressional Research Service Report. Washington.

Taylor, L. (2017). Sendero Luminoso in the New Millennium: Comrades, Cocaine and Counter-Insurgency on the Peruvian Frontier. Journal of Agrarian Change, 17(1), 106-121. https://doi.org/10.1111/joac.12137

UNODC. (2017). Informe de Monitoreo de Cultivos de coca en Perú. In Oficina de las Naciones Unidas contra la Droga y el Delito.

Uvin, P. (2001). Difficult choices in the new post-conflict agenda: The international community in Rwanda after the genocide. Third World Quarterly, 22(2), 177-189. https://doi.org/10.1080/01436590120043291 
